\title{
On a problem of Erdős and Graham concerning digits
}

\author{
by \\ Thomas Stoll (Wien)
}

1. Introduction. Let $m \in \mathbb{Z}^{+}$and consider the sequence of positive integers $\left(u_{n}\right)_{n \geq 1}$ defined by

$$
u_{1}=m, \quad u_{n+1}=\left\lfloor\sqrt{2}\left(u_{n}+1 / 2\right)\right\rfloor,
$$

which originates from work of F. K. Hwang and S. Lin on Ford and Johnson's sorting algorithm [7]. In a short note, R. L. Graham and H. O. Pollak [5] provided an explicit expression for $u_{n}$, namely, $u_{n}=\left\lfloor\tau\left(2^{(n-1) / 2}+2^{(n-2) / 2}\right)\right\rfloor$, $n \geq 2$, where $\tau$ is the $m$ th smallest real number in the set $\{1,2,3, \ldots\} \cup$ $\{\sqrt{2}, 2 \sqrt{2}, 3 \sqrt{2}, \ldots\}$. From this, they noticed the following unexpected fact, which is the hub of the present article.

FACT 1 (Graham-Pollak). If $m=1$, then

$$
d_{n}=u_{2 n+1}-2 u_{2 n-1}
$$

is the $n$th binary digit of $\sqrt{2}=(1.011010100 \ldots)_{2}$.

This curious result has been cited several times, for instance, by P. Erdős and R. L. Graham [2, p. 96], by R. K. Guy [6, Ex. 30], by R. L. Graham, D. E. Knuth and O. Patashnik [4, Ex. 3.46] and-more recently-by J. Borwein and D. Bailey $\left({ }^{1}\right)[1$, p. 62-63]. N. J. A. Sloane's online encyclopedia of integer sequences [9] includes eight sequences which are related to GrahamPollak's sequence (1.1). However, it is not obvious from Graham-Pollak's

2000 Mathematics Subject Classification: Primary 11B37; Secondary 11A63.

Key words and phrases: Graham-Pollak's sequence, digital expansions.

Research supported by the Austrian Science Foundation (FWF), project S9604, "Analytic and Probabilistic Methods in Combinatorics".

$\left({ }^{1}\right)$ Therein, the authors erroneously refer for details to J. V. Grabiner, Is Mathematical Truth Time-Dependent?, in: New Directions in the Philosophy of Mathematics, Th. Tymoczko (ed.), Princeton Univ. Press, 1998. But Graham-Pollak's sequence is not mentioned there. 
proof how to generalize this singular result. In the closing paragraph of Chapter 9 of [2], "Miscellaneous Problems", Erdős and Graham suspected that

"there must be similar results for $\sqrt{\alpha}$ and other algebraic numbers but we have no idea what they are".

The main goal of the present exposition is to vastly extend Fact 1 to multi-parametric instances of recurrences of type (1.1). Partial results on this "unconventional problem" [2] have been obtained by S. Rabinowitz and P. Gilbert [8] and the author [10], in both cases giving an infinite number of recurrences which incorporate Fact 1 . Regarding our main results (Theorems $3.1,3.3$ and 3.4), we are able to replace $\sqrt{2}$ by $w \in \mathbb{R}^{+}, 1 / 2$ by $\varepsilon \in \mathbb{R}$ and to introduce families of recurrences, which involve three new parameters $m, l, k \in \mathbb{Z}$ as well as allow digital expansions with respect to any base $g \geq 2$.

The paper is organized as follows. In Section 2 we introduce the set of triples $(m, l, k)$ for which we establish infinitely many recurrences in Theorem 3.1. By specializing, we obtain new curious examples.

EXAmple 1.1. Define the sequence $\left(v_{n}\right)_{n \geq 1}$ by

$$
v_{1}=3, \quad v_{n+1}= \begin{cases}\left\lfloor-\frac{3}{e+9}\left(v_{n}+\pi\right)\right\rfloor & \text { if } n \text { is odd } \\ \left\lfloor-(e+9)\left(v_{n}+1\right)\right\rfloor & \text { if } n \text { is even. }\end{cases}
$$

Then the number $v_{2 n+1}-3 v_{2 n-1}$ is the $n$th ternary digit of $e=\exp (1)=$ $(2.201101121 \ldots)_{3}$.

In Section 3 we separately treat the binary case $g=2$ (cf. Theorems 3.3 and 3.4), where we find two additional families of floor recurrences. Plugging in $w=\sqrt{2}, \varepsilon=1 / 2$ and $(m, l, k)=(1,0,0)$ in Theorem 3.3, we reobtain Graham-Pollak's result. More generally, we join Theorems 3.3 and 3.4 with Beatty's theorem to show that (1.1) gives rise to binary digits for all $m \in \mathbb{Z}$. Corollary 3.5 characterizes all represented numbers and thus unifies the examples listed by Borwein and Bailey [1] for $1 \leq m \leq 10$. Section 4 is devoted to the proofs of the three main results and of Corollary 3.5, which are based on inductive arguments.

2. Notation. Let $g \in \mathbb{Z}, g \geq 2$ and $w \in \mathbb{R}^{+}$with $w=\sum_{i=1}^{\infty} d_{i} g^{M-i+1}$ its unique base $g$ expansion, i.e., $d_{i} \in \mathbb{Z}$ with $0 \leq d_{i}<g$ and $d_{1} \neq 0$. Further, let $M=\left\lfloor\log _{g} w\right\rfloor$ and $t=w / g^{M}$. Then $t=\left(d_{1} \cdot d_{2} d_{3} \ldots\right)_{g}$ with $1 \leq t<g$, thus there is no need to distinguish between the digits of $w$ and the digits of $t$. In what follows, we will often use $t$ as the normalized version of $w$. 
Definition 2.1. Let $\Omega=\Omega_{1} \cup \Omega_{2}$ with

$$
\begin{aligned}
& \Omega_{1}=\left\{(m, l) \in \mathbb{Z} \times \mathbb{Z} \backslash\{0\} \mid m \geq 1,-\frac{m g+1}{2 g-1}<l<\frac{m g+g}{2 g-1}\right\}, \\
& \Omega_{2}=\left\{(m, l) \in \mathbb{Z} \times \mathbb{Z} \backslash\{0\} \mid m \leq-2, \frac{m g+g}{2 g-1}<l<-\frac{m g+1}{2 g-1}\right\} .
\end{aligned}
$$

In view of Theorem 3.1, the set $\Omega$ describes all pairs $(m, l)$ for which we give at least one recurrence of type (1.1) yielding $g$-ary digits. Since the bounds appearing in the definition of $\Omega_{1}$ and $\Omega_{2}$ are linear, the set $\Omega$ describes the union of two infinite cones. Concerning the total number of recurrences attached to one such pair $(m, l)$, we need to split $\Omega_{1}$ and $\Omega_{2}$ up into a total of six subsets (subcones).

Definition 2.2. Let $\Omega_{1}=\mathcal{A}_{1} \cup \mathcal{A}_{2} \cup \mathcal{A}_{3}$ and $\Omega_{2}=\mathcal{A}_{4} \cup \mathcal{A}_{5} \cup \mathcal{A}_{6}$ with

$$
\begin{array}{ll}
\mathcal{A}_{1}=\left\{(m, l) \in \Omega_{1} \mid l<0\right\}, & \mathcal{A}_{4}=\left\{(m, l) \in \Omega_{2} \mid l<0\right\}, \\
\mathcal{A}_{2}=\left\{(m, l) \in \Omega_{1} \mid 0<l \leq g-1\right\}, & \mathcal{A}_{5}=\left\{(m, l) \in \Omega_{2} \mid 0<l \leq g-1\right\}, \\
\mathcal{A}_{3}=\left\{(m, l) \in \Omega_{1} \mid l>g-1\right\}, & \mathcal{A}_{6}=\left\{(m, l) \in \Omega_{2} \mid l>g-1\right\} .
\end{array}
$$

To each $(m, l) \in \mathcal{A}_{i}$ we introduce a third parameter $k \in \mathbb{Z}$, which is taken from a certain interval depending on $1 \leq i \leq 6$. Note that by the linear constraints in Definition 2.1, for any $(m, l) \in \mathcal{A}_{i}$ we have $(m, l, \pm 1) \in \mathcal{D}_{i}$.

Definition 2.3. For $1 \leq i \leq 6$ set

$$
\mathcal{D}_{i}=\left\{(m, l, k)\left|(m, l) \in \mathcal{A}_{i}, 0<\right| k \mid<\beta_{i}, k \in \mathbb{Z}\right\},
$$

with

$$
\begin{array}{ll}
\beta_{1}=-\beta_{6}=-\frac{(m g+l+1)(g-1)}{l g}, & \beta_{2}=\frac{(m g+1)(g-1)}{l g}, \\
\beta_{3}=-\beta_{4}=\frac{(m g+g-l)(g-1)}{l g}, & \beta_{5}=-\frac{(m-1)(g-1)}{l} .
\end{array}
$$

Furthermore, set $\mathcal{D}_{i}=\mathcal{D}_{i}^{+} \cup \mathcal{D}_{i}^{-}$with $\mathcal{D}_{i}^{+}=\left\{(m, l, k) \mid(m, l, k) \in \mathcal{D}_{i}, k>0\right\}$ and $\mathcal{D}_{i}^{-}=\left\{(m, l, k) \mid(m, l, k) \in \mathcal{D}_{i}, k<0\right\}$.

The next definition is included in order to state the general main result in a concise form. Basically, to each $(m, l, k) \in \mathcal{D}_{i}^{+}$(resp. $\left.\mathcal{D}_{i}^{-}\right)$we attach numbers $\gamma_{i}^{+}, \delta_{i}^{+}$(resp. $\gamma_{i}^{-}, \delta_{i}^{-}$) which build up the interval for $\varepsilon$ in the recurrence of Theorem 3.1. It is a straightforward calculation from Definition 2.3 that this interval is non-empty, i.e., $1+\gamma_{i}^{+}<\delta_{i}^{+}$(resp. $\left.1+\gamma_{i}^{-}<\delta_{i}^{-}\right)$if $(m, l, k) \in \mathcal{D}_{i}^{+}\left(\operatorname{resp} . \mathcal{D}_{i}^{-}\right)$. 
Definition 2.4. Let $(m, l, k) \in \mathcal{D}_{i}$ and $\gamma_{i}^{+}, \gamma_{i}^{-}, \delta_{i}^{+}, \delta_{i}^{-} \in \mathbb{R}, 1 \leq i \leq 6$, with

$$
\begin{aligned}
& \gamma_{2}^{+}=\delta_{2}^{-}=\gamma_{3}^{+}=\delta_{3}^{-}=\gamma_{4}^{+}=\delta_{4}^{-}=-\frac{m g+1}{k g}, \\
& \delta_{2}^{+}=\gamma_{2}^{-}=\gamma_{1}^{+}=\delta_{1}^{-}=\gamma_{6}^{+}=\delta_{6}^{-}=\frac{g-l-1}{k l g}(m g+1), \\
& \delta_{5}^{+}=\gamma_{5}^{-}=\delta_{1}^{+}=\gamma_{1}^{-}=\delta_{6}^{+}=\gamma_{6}^{-}=-\frac{m+1}{k}, \\
& \gamma_{5}^{+}=\delta_{5}^{-}=\delta_{3}^{+}=\gamma_{3}^{-}=\delta_{4}^{+}=\gamma_{4}^{-}=\frac{g-l-1}{k l}(m+1) .
\end{aligned}
$$

3. Main results. Our general main result is

Theorem 3.1. Let $w \in \mathbb{R}^{+}, g \in \mathbb{Z}, g \geq 2$ and $t=w / g^{M}$, where $M=$ $\left\lfloor\log _{g} w\right\rfloor$. Furthermore, let $(m, l, k) \in \mathcal{D}_{i}^{+}$(resp. $\left.\mathcal{D}_{i}^{-}\right)$for some $1 \leq i \leq 6$ with $(g-1) \mid(k-1) l$. Define the sequence $\left(u_{n}\right)_{n \geq 1}$ by

$$
u_{1}=m, \quad u_{n+1}= \begin{cases}\left\lfloor a\left(u_{n}+\varepsilon\right)\right\rfloor & \text { if } n \text { is odd, } \\ \left\lfloor b\left(u_{n}+l /(g-1)\right)\right\rfloor & \text { if } n \text { is even }\end{cases}
$$

where

$$
a=\frac{k l g}{(g-1)(t+m g)}, \quad b=\frac{g}{a},
$$

and $1+\gamma_{i}^{+} \leq \varepsilon<\delta_{i}^{+}$(resp. $\left.1+\gamma_{i}^{-}<\varepsilon \leq \delta_{i}^{-}\right)$. Then $u_{2 n+1}-g u_{2 n-1}$ is the $n$th digit in the $g$-ary expansion of $w$.

For illustration, we start with an easy but striking example. Let $g=3$, $m=3$ and $l=2$. Then $(3,2) \in \mathcal{A}_{2} \subset \Omega_{1}$ and $\beta_{2}=10 / 3$ and $\{(3,2, \pm 1)$, $(3,2, \pm 2),(3,2, \pm 3)\} \subset \mathcal{D}_{2}$. Note that $(3,2,1),(3,2,2),(3,2,3) \in \mathcal{D}_{2}^{+}$and $(3,2,-1),(3,2,-2),(3,2,-3) \in \mathcal{D}_{2}^{-}$, and that each of these six triples satisfies $(g-1) \mid(k-1) l$. Thus, according to Theorem 3.1, there are six different recurrences yielding ternary digits for $(m, l)=(3,2)$. For instance, take the triple $(3,2,-1) \in \mathcal{D}_{2}^{-}$. Then $1+\gamma_{2}^{-}=1$ and $\delta_{2}^{-}=10 / 3$, and for $w=t=e=\exp (1)$ and $\varepsilon=\pi$ we get the result mentioned in Example 1.1.

Unfortunately, whatever parameters one chooses in Theorem 3.1, it is not possible to merge the two cases corresponding to the parity of $n$. Despite this fact, we can at least afford that $l /(g-1)=\varepsilon=1 / 2$, thus giving a version of Fact 1 for odd bases $g \geq 3$. For that purpose, observe that $l=(g-1) / 2$ implies $(m, l, 1) \in \mathcal{D}_{2}^{+}$if $m \geq 1$, resp. $(m, l, 1) \in \mathcal{D}_{5}^{+}$if $m \leq-2$. In more explicit terms, we have the following result.

Corollary 3.2. Let $w \in \mathbb{R}^{+}, g, m \in \mathbb{Z}, g \geq 3$ odd, $m \notin\{-1,0\}$ and $t=w / g^{M}$, where $M=\left\lfloor\log _{g} w\right\rfloor$. Define the sequence $\left(v_{n}\right)_{n \geq 0}$ by

$$
v_{1}=m, \quad v_{n+1}=\left\lfloor c_{n+1}\left(v_{n}+1 / 2\right)\right\rfloor,
$$


where

$$
c_{n+1}= \begin{cases}(2(t+m g))^{-1} g & \text { if } n \text { is odd, } \\ 2(t+m g) & \text { if } n \text { is even. }\end{cases}
$$

Then $v_{2 n+1}-g v_{2 n-1}$ is the $n$th digit in the $g$-ary expansion of $w$.

The next two results (Theorems 3.3 and 3.4) give two additional families of recurrences for expansions with respect to base $g=2$, which are not covered by Theorem 3.1. These families are of a different nature, and cannot be obtained by plainly shifting $n \mapsto n+1$. Observe also that the bounds for $\varepsilon$ in Theorem 3.3 are independent of $k$, whereas those in Theorem 3.4 are not.

ThEOREM 3.3. Let $w \in \mathbb{R}^{+}$and $t=w / 2^{M}=\left(d_{1} \cdot d_{2} d_{3} \ldots\right)_{2}$, where $M=\left\lfloor\log _{2} w\right\rfloor$. Furthermore, let $m, l, k \in \mathbb{Z}$ with $m \notin\{-1,0\}, k \geq 0$ and $0 \leq l \leq m-1$ if $m \geq 1$, resp. $m+1 \leq l \leq-1$ if $m \leq-2$. Define the sequence $\left(u_{n}\right)_{n \geq 1}$ by

$$
u_{1}=m, \quad u_{n+1}= \begin{cases}\left\lfloor a\left(u_{n}+1 / 2\right)\right\rfloor & \text { if } n \text { is odd }, \\ \left\lfloor b\left(u_{n}+\varepsilon\right)\right\rfloor & \text { if } n \text { is even },\end{cases}
$$

where

$$
a=2 k+1+\frac{t+2 l}{t+2 m}, \quad b=\frac{2}{a},
$$

and

$$
\begin{array}{cl}
\frac{1}{2}-\frac{2 l+1}{2(2 m+1)} \leq \varepsilon<\frac{1}{2}+\frac{2 l+1}{2(2 m+1)} & \text { if } m \geq 1, \\
\frac{1}{2}-\frac{l+1}{2(m+1)} \leq \varepsilon \leq \frac{1}{2}+\frac{l+1}{2(m+1)} & \text { if } m \leq-2 .
\end{array}
$$

Then $u_{2 n+1}-2 u_{2 n-1}=d_{n}$ and $u_{2 n+2}-2 u_{2 n}=d_{n+1}+k\left(2 d_{n}-1\right)$.

If $w=\sqrt{2}$ and $(m, l, k)=(1,0,0)$ then $a=b=\sqrt{2}$ and with $\varepsilon=1 / 2$ we retrieve Graham-Pollak's result for the binary digits of $\sqrt{2}$. In fact, these digits are obtained whenever $1 / 3 \leq \varepsilon<2 / 3$.

ThEOREM 3.4. Let $w \in \mathbb{R}^{+}$and $t=w / 2^{M}=\left(d_{1} \cdot d_{2} d_{3} \ldots\right)_{2}$, where $M=\left\lfloor\log _{2} w\right\rfloor$. Furthermore, let $m, l, k \in \mathbb{Z}$ with $m \notin\{-1,0\}, k \geq 0$ and $1 \leq l \leq m$ if $m \geq 1$, resp. $m+1 \leq l \leq-1$ if $m \leq-2$. Define the sequence $\left(u_{n}\right)_{n \geq 1}$ by

$$
u_{1}=m, \quad u_{n+1}= \begin{cases}\left\lfloor a\left(u_{n}+\varepsilon\right)\right\rfloor & \text { if } n \text { is odd }, \\ \left\lfloor b\left(u_{n}+1 / 2\right)\right\rfloor & \text { if } n \text { is even }\end{cases}
$$

where

$$
a=2 k+1+\frac{2 l}{t+2 m}, \quad b=\frac{2}{a},
$$


and

$$
\begin{array}{ll}
\frac{1}{2}-\frac{m-l+1 / 2}{(2 k+1)(2 m+1)+2 l} \leq \varepsilon<\frac{1}{2}+\frac{m-l+1 / 2}{(2 k+1)(2 m+1)+2 l} & \text { if } m \geq 1, \\
\frac{1}{2}-\frac{m-l+1}{2(2 k+1)(m+1)+2 l} \leq \varepsilon \leq \frac{1}{2}+\frac{m-l+1}{2(2 k+1)(m+1)+2 l} & \text { if } m \leq-2 .
\end{array}
$$

Then $u_{2 n+1}-2 u_{2 n-1}=d_{n}$ and $u_{2 n+2}-2 u_{2 n}=d_{n}+k\left(2 d_{n}-1\right)$.

For both families, in plain contrast to Theorem 3.1, it is possible to merge the two cases corresponding to the parity of $n$, namely, an infinite number of times. Indeed, we can use Theorems 3.3 and 3.4 together with a suitable normalization to retrieve a generalization of Fact 1 for all $m \in \mathbb{Z} \backslash\{-1,0\}$. Concerning (1.1), both cases $m=0$ and $m=-1$ yield only trivial sequences since $u_{2 n+1}-2 u_{2 n-1} \equiv 0$ if $m=0$, resp., $u_{2 n+1}-2 u_{2 n-1} \equiv 1$ if $m=-1$.

To begin with, define $S(\alpha)=\{\lfloor r \alpha\rfloor \mid r \in \mathbb{Z}\}, \alpha \in \mathbb{R}$. Since $(1+\sqrt{2})^{-1}+$ $(1+1 / \sqrt{2})^{-1}=1$ and $1+\sqrt{2} \in \mathbb{R} \backslash \mathbb{Q}$ it is immediate from Beatty's theorem (cf. [3]) that $S(1+1 / \sqrt{2}) \cup S(1+\sqrt{2})=\mathbb{Z} \backslash\{-1\}$ and $S(1+1 / \sqrt{2}) \cap S(1+\sqrt{2})$ $=\{0\}$. Therefore, for any $m \in \mathbb{Z} \backslash\{-1,0\}$ there is a unique $r \in \mathbb{Z}$ such that either $m=\lfloor r(1+1 / \sqrt{2})\rfloor$ or $m=\lfloor r(1+\sqrt{2})\rfloor$.

Corollary 3.5. Let $m \in \mathbb{Z} \backslash\{-1,0\}$ and set

$$
w= \begin{cases}r \sqrt{2}-2\lfloor r / \sqrt{2}\rfloor & \text { if } m=\lfloor r(1+1 / \sqrt{2})\rfloor, r \in \mathbb{Z}, \\ 2 r \sqrt{2}-2\lfloor r \sqrt{2}\rfloor & \text { if } m=\lfloor r(1+\sqrt{2})\rfloor, r \in \mathbb{Z},\end{cases}
$$

with $M=\left\lfloor\log _{2} w\right\rfloor$. Define the sequence $\left(u_{n}\right)_{n \geq 1}$ by

$$
u_{1}=m, \quad u_{n+1}=\left\lfloor\sqrt{2}\left(u_{n}+1 / 2\right)\right\rfloor .
$$

Then $u_{2(n-M)+1}-2 u_{2(n-M)-1}$ denotes the $n$th binary digit of $w$.

We similarly derive from Theorems 3.3 and 3.4 that for all $m \in \mathbb{Z} \backslash\{-1,0\}$ the quantity $u_{2(n-M)+2}-2 u_{2(n-M)}$ defines the $n$th binary digit of $w=$ $2 r \sqrt{2}-2\lfloor r \sqrt{2}\rfloor, M=\left\lfloor\log _{2} w\right\rfloor$. This is a closed-form expression for the examples given by Borwein and Bailey [1] and by Sloane [9] (A091524, A091525):

\begin{tabular}{c|ccccc}
\hline$m$ & 1 & 2 & 3 & 4 & 5 \\
$w / 2$ & $\sqrt{2}-1$ & $\sqrt{2}-1$ & $2 \sqrt{2}-2$ & $2 \sqrt{2}-2$ & $3 \sqrt{2}-4$ \\
\hline & \multicolumn{5}{|c}{} \\
\hline$m$ & 6 & 7 & 8 & 9 & 10 \\
$w / 2$ & $4 \sqrt{2}-5$ & $3 \sqrt{2}-4$ & $5 \sqrt{2}-7$ & $4 \sqrt{2}-5$ & $6 \sqrt{2}-8$ \\
\hline
\end{tabular}

4. Proofs. First, as an auxiliary result, we point out an explicit expression for $u_{2 n+1}$, provided $u_{2 n+1}-g u_{2 n-1}$ denotes $g$-ary digits. 
Proposition 4.1. Let $w \in \mathbb{R}^{+}$with $0<w<g$ and put $t=w g^{-M}=$ $\left(d_{1} . d_{2} d_{3} \ldots\right)_{g}$ where $M=\left\lfloor\log _{g} w\right\rfloor$. Moreover, let $m \in \mathbb{Z}$ and define $\left(u_{n}\right)_{n \geq 1}$ by $u_{1}=m$ and

$$
u_{2 n+1}=g u_{2 n-1}+ \begin{cases}0 & \text { if } 1 \leq n \leq-M \\ d_{n+M} & \text { if } n>-M\end{cases}
$$

Then $u_{2 n+1}=m g^{n}+\left\lfloor w g^{n-1}\right\rfloor$ and $u_{2(n-M)+1}-g u_{2(n-M-1)+1}=d_{n}$.

Proof. Since $u_{2 n+1}=m g^{n}+\sum_{i=1}^{n+M} d_{i} g^{n+M-i}$ the statement follows from

$$
\begin{aligned}
u_{2(n-M)+1}-g u_{2(n-M-1)+1} & =d_{n}=\left(d_{1} d_{2} \ldots d_{n}\right)_{g}-\left(d_{1} d_{2} \ldots d_{n-1} 0\right)_{g} \\
& =\left\lfloor t g^{n-1}\right\rfloor-g\left\lfloor t g^{n-2}\right\rfloor .
\end{aligned}
$$

4.1. Proof of Theorem 3.1. We claim $u_{2 n}=l\left(k g^{n-1}-1\right) /(g-1)$ and $u_{2 n+1}=m g^{n}+\left\lfloor t g^{n-1}\right\rfloor$, the latter being a necessary condition by Proposition 4.1. Since $1 \leq t<g$ we have $u_{1}=m g^{0}+\lfloor t / g\rfloor=m$. By induction suppose first that the result holds for $u_{2 n}$. Then

$$
u_{2 n+1}=\left\lfloor b\left(u_{2 n}+l /(g-1)\right)\right\rfloor=\left\lfloor(t+m g) g^{n-1}\right\rfloor=m g^{n}+\left\lfloor t g^{n-1}\right\rfloor .
$$

Assume now that the result holds for $u_{2 n+1}$. Then

$$
\begin{aligned}
u_{2 n+2} & =\left\lfloor a\left\lfloor(t+m g) g^{n-1}\right\rfloor+a \varepsilon\right\rfloor=\left\lfloor a\left\lfloor\frac{k l g^{n}}{a(g-1)}\right\rfloor+a \varepsilon\right\rfloor \\
& =l \frac{k g^{n}-1}{g-1}+\left\lfloor\frac{l}{g-1}-a\left\{\frac{k l g^{n}}{a(g-1)}\right\}+a \varepsilon\right\rfloor,
\end{aligned}
$$

where $\{x\}$ denotes the (positive) fractional part of $x \in \mathbb{R}$ and where we have $l\left(k g^{n}-1\right) /(g-1)=l k\left(g^{n}-1\right) /(g-1)+l(k-1) /(g-1) \in \mathbb{Z}$. It remains to ensure that for all $1 \leq t<g$,

$$
0 \leq \frac{l}{g-1}-a\left\{\frac{k l g^{n}}{a(g-1)}\right\}+a \varepsilon<1 .
$$

We distinguish several cases.

First, let $(m, l, k) \in \mathcal{D}_{1}^{-} \cup \mathcal{D}_{2}^{+} \cup \mathcal{D}_{3}^{+}$. Then $a>0$ with

$$
a \in\left(\frac{k l}{(1+m)(g-1)}, \frac{k l g}{(1+m g)(g-1)}\right]=: I_{1} .
$$

Condition (4.1) holds if we can guarantee that

$$
l /(g-1)+a \varepsilon<1 \text { and } l /(g-1)+a(\varepsilon-1) \geq 0
$$

for all $1 \leq t<g$. Hence, it suffices to ensure that

$$
\min _{a \in I_{1}}\left(\frac{1}{a}-\frac{l}{a(g-1)}\right)>\varepsilon \geq \max _{a \in I_{1}}\left(1-\frac{l}{a(g-1)}\right) .
$$


For $(m, l, k) \in \mathcal{D}_{1}^{-}$we obtain

$$
\frac{(1+m g)(g-1)}{k l g}\left(1-\frac{l}{g-1}\right)>\varepsilon \geq 1-\frac{l}{g-1} \cdot \frac{(1+m)(g-1)}{k l},
$$

which is equivalent to $1+\gamma_{1}^{-} \leq \varepsilon<\delta_{1}^{-}$. Similarly, for $(m, l, k) \in \mathcal{D}_{2}^{+}$, condition (4.3) translates into

$$
\frac{(1+m g)(g-1)}{k l g}\left(1-\frac{l}{g-1}\right)>\varepsilon \geq 1-\frac{l}{g-1} \cdot \frac{(1+m g)(g-1)}{k l g},
$$

which is $1+\gamma_{2}^{+} \leq \varepsilon<\delta_{2}^{+}$. Finally, if $(m, l, k) \in \mathcal{D}_{3}^{+}$, then

$$
\frac{(1+m)(g-1)}{k l}\left(1-\frac{l}{g-1}\right)>\varepsilon \geq 1-\frac{l}{g-1} \cdot \frac{(1+m g)(g-1)}{k l g},
$$

thus $1+\gamma_{3}^{+} \leq \varepsilon<\delta_{3}^{+}$. Now, let $(m, l, k) \in \mathcal{D}_{4}^{+} \cup \mathcal{D}_{5}^{-} \cup \mathcal{D}_{6}^{-}$. Then $a>0$ as well, with

$$
a \in\left(\frac{k l g}{(1+m g)(g-1)}, \frac{k l}{(1+m)(g-1)}\right]=: I_{2},
$$

where $I_{2}$ has reversed endpoints with respect to $I_{1}$. Using the above calculations we get $1+\gamma_{6}^{-} \leq \varepsilon<\delta_{6}^{-}, 1+\gamma_{4}^{+} \leq \varepsilon<\delta_{4}^{+}, 1+\gamma_{5}^{-} \leq \varepsilon<\delta_{5}^{-}$with $\gamma_{6}^{-}=\gamma_{1}^{-}, \delta_{6}^{-}=\delta_{1}^{-}, \gamma_{4}^{+}=\gamma_{3}^{+}, \delta_{4}^{+}=\delta_{3}^{+}$and $\gamma_{5}^{-}=\gamma_{1}^{-}, \delta_{5}^{-}=\delta_{3}^{+}$.

Secondly, let $(m, l, k) \in \mathcal{D}_{1}^{+} \cup \mathcal{D}_{2}^{-} \cup \mathcal{D}_{3}^{-}$. Then $a<0$ with $a \in I_{2}$ and it is sufficient to show that

$$
0 \leq l /(g-1)+a \varepsilon \text { and } l /(g-1)+a(\varepsilon-1)<1
$$

for all $1 \leq t<g$. We ensure that

$$
\min _{a \in I_{2}}\left(-\frac{l}{a(g-1)}\right) \geq \varepsilon>1+\max _{a \in I_{2}}\left(\frac{1}{a}-\frac{l}{a(g-1)}\right) .
$$

For $(m, l, k) \in \mathcal{D}_{1}^{+}$we have

$$
-\frac{(1+m)(g-1)}{k l} \cdot \frac{l}{g-1} \geq \varepsilon>\left(1-\frac{l}{g-1}\right) \cdot \frac{(1+m g)(g-1)}{k l g}+1,
$$

which is equivalent to $1+\gamma_{1}^{+}<\varepsilon \leq \delta_{1}^{+}$. If $(m, l, k) \in \mathcal{D}_{2}^{-}$then

$$
-\frac{(1+m g)(g-1)}{k l g} \cdot \frac{l}{g-1} \geq \varepsilon>\left(1-\frac{l}{g-1}\right) \cdot \frac{(1+m g)(g-1)}{k l g}+1,
$$

and $1+\gamma_{2}^{-}<\varepsilon \leq \delta_{2}^{-}$. If $(m, l, k) \in \mathcal{D}_{3}^{-}$, then

$$
-\frac{(1+m g)(g-1)}{k l g} \cdot \frac{l}{g-1} \geq \varepsilon>\left(1-\frac{l}{g-1}\right) \cdot \frac{(1+m)(g-1)}{k l}+1,
$$

and $1+\gamma_{3}^{-}<\varepsilon \leq \delta_{3}^{-}$. Finally, consider $(m, l, k) \in \mathcal{D}_{4}^{-} \cup \mathcal{D}_{5}^{+} \cup \mathcal{D}_{6}^{+}$. Then $a<0$ with $a \in I_{1}$ and the above calculations yield $1+\gamma_{5}^{+} \leq \varepsilon<\delta_{5}^{+}$, $1+\gamma_{6}^{+} \leq \varepsilon<\delta_{6}^{+}, 1+\gamma_{4}^{-} \leq \varepsilon<\delta_{4}^{-}$with $\gamma_{5}^{+}=\gamma_{3}^{-}, \delta_{5}^{+}=\delta_{1}^{+}, \gamma_{6}^{+}=\gamma_{2}^{-}$, $\delta_{6}^{+}=\delta_{1}^{+}$and $\gamma_{4}^{-}=\gamma_{3}^{-}, \delta_{4}^{-}=\delta_{2}^{-}$. This completes the proof of Theorem 3.1. 
4.2. Proof of Theorems 3.3 and 3.4 and Corollary 3.5

Proof of Theorem 3.3. By Proposition 4.1 it suffices to prove that for $n \geq 1$

$$
\begin{aligned}
u_{2 n-1} & =m 2^{n-1}+\left\lfloor t 2^{n-2}\right\rfloor, \\
u_{2 n} & =(m+l) 2^{n-1}+\left\lfloor t 2^{n-1}\right\rfloor+k\left(m 2^{n}+2\left\lfloor t 2^{n-2}\right\rfloor+1\right) .
\end{aligned}
$$

Since $1 \leq t<2$, we have $u_{1}=m+\lfloor t / 2\rfloor=m$. By induction, assume (4.5). Then

$$
\begin{aligned}
u_{2 n} & =\left\lfloor\left(2 k+1+\frac{t+2 l}{t+2 m}\right)\left(m 2^{n-1}+\left\lfloor t 2^{n-2}\right\rfloor+\frac{1}{2}\right)\right\rfloor \\
& =k\left(m 2^{n}+2\left\lfloor t 2^{n-2}\right\rfloor+1\right)+\left\lfloor\frac{t+m+l}{t+2 m}\left(m 2^{n}+2\left\lfloor t 2^{n-2}\right\rfloor+1\right)\right\rfloor .
\end{aligned}
$$

Thus, it is sufficient to ensure that

$$
\begin{aligned}
(m+l) 2^{n-1}+\left\lfloor t 2^{n-1}\right\rfloor & \leq \frac{t+m+l}{t+2 m}\left(m 2^{n}+2\left\lfloor t 2^{n-2}\right\rfloor+1\right) \\
& <(m+l) 2^{n-1}+\left\lfloor t 2^{n-1}\right\rfloor+1
\end{aligned}
$$

for all $1 \leq t<2$. First, let $m \geq 1$, thus $t+2 m>0$. Then by using $2\left\lfloor t 2^{n-2}\right\rfloor=\left\lfloor t 2^{n-1}\right\rfloor-d_{n}$ we rewrite (4.7) in the form

$$
\begin{aligned}
l t 2^{n-1}+m\left\lfloor t 2^{n-1}\right\rfloor & \leq m t 2^{n-1}+l\left\lfloor t 2^{n-1}\right\rfloor+(t+m+l)\left(1-d_{n}\right) \\
& <l t 2^{n-1}+m\left\lfloor t 2^{n-1}\right\rfloor+t+2 m .
\end{aligned}
$$

Hence,

$$
0 \leq(m-l)\left(t 2^{n-1}-\left\lfloor t 2^{n-1}\right\rfloor\right)+(t+m+l)\left(1-d_{n}\right)<t+2 m,
$$

which is true since $1 \leq m-l, t 2^{n-1}-\left\lfloor t 2^{n-1}\right\rfloor \in[0,1)$ and $1-d_{n} \in\{0,1\}$. By the same reasoning we show that for $m \leq-2$ and $m+1 \leq l \leq-1$ we have

$$
0 \geq(m-l)\left(t 2^{n-1}-\left\lfloor t 2^{n-1}\right\rfloor\right)+(t+m+l)\left(1-d_{n}\right)>t+2 m .
$$

Now, suppose (4.6). Then we have to show that

$$
u_{2 n+1}=\left\lfloor\frac{2(t+2 m)}{(2 k+1)(t+2 m)+t+2 l}\left(u_{2 n}+\varepsilon\right)\right\rfloor,
$$

or equivalently,

$$
\begin{aligned}
m 2^{n} & +\left\lfloor t 2^{n-1}\right\rfloor \\
& \leq \frac{2(t+2 m)\left((m+l) 2^{n-1}+\left\lfloor t 2^{n-1}\right\rfloor+k\left(m 2^{n}+2\left\lfloor t 2^{n-2}\right\rfloor+1\right)+\varepsilon\right)}{(2 k+1)(t+2 m)+t+2 l} \\
& <m 2^{n}+\left\lfloor t 2^{n-1}\right\rfloor+1 .
\end{aligned}
$$


First, let $m \geq 1$. Then the denominator of the middle term is positive and straightforward algebraic manipulation yields

$$
\begin{aligned}
2(t+ & 2 m) k\left\lfloor t 2^{n-1}\right\rfloor+m t 2^{n}+2 l\left\lfloor t 2^{n-1}\right\rfloor \\
& \leq 2(t+2 m)\left(2 k\left\lfloor t 2^{n-2}\right\rfloor+k+\varepsilon\right)+l t 2^{n}+2 m\left\lfloor t 2^{n-1}\right\rfloor \\
& <2(t+2 m) k\left\lfloor t 2^{n-1}\right\rfloor+m t 2^{n}+2 l\left\lfloor t 2^{n-1}\right\rfloor+(2 k+1)(t+2 m)+t+2 l .
\end{aligned}
$$

Again, plugging in $2\left\lfloor t 2^{n-2}\right\rfloor=\left\lfloor t 2^{n-1}\right\rfloor-d_{n}$, we obtain

$$
\begin{aligned}
0 & \leq 2(t+2 m)\left(k\left(1-d_{n}\right)+\varepsilon\right)+(m-l)\left(2\left\lfloor t 2^{n-1}\right\rfloor-t 2^{n}\right) \\
& <2(t+2 m)(k+1)-2(m-l) .
\end{aligned}
$$

We now consider both inequalities of (4.8) separately. The right-hand side inequality gives

$$
2(t+2 m)\left(\varepsilon-k d_{n}-1\right)+(m-l) \xi<0,
$$

where $\xi=2\left\lfloor t 2^{n-1}\right\rfloor-t 2^{n}+2 \in(0,2]$. Then

$$
\varepsilon<\frac{m+l+1}{2 m+1}=1-\frac{(m-l) \cdot 2}{2(1+2 m)} \leq 1+k d_{n}-\frac{(m-l) \xi}{2(t+2 m)},
$$

thus (4.9) holds for $1 \leq t<2$. For the left-hand side inequality in (4.8), put $\xi^{\prime}=2\left\lfloor t 2^{n-1}\right\rfloor-t 2^{n} \in(-2,0]$. Then

$$
0 \leq 2(t+2 m)\left(\varepsilon+k-k d_{n}\right)+(m-l) \xi^{\prime}
$$

and

$$
-\frac{(m-l) \xi^{\prime}}{2(t+2 m)}+k\left(d_{n}-1\right)<-\frac{(m-l) \cdot(-2)}{2(1+2 m)} \leq \varepsilon .
$$

This completes the induction step for $m \geq 1$.

Now, suppose $m \leq-2$ and $m-l<0$. Then also $(2 k+1)(t+2 m)+t+2 l<0$ and $t+2 m<0$, thus

$$
-\frac{(m-l) \xi^{\prime}}{2(t+2 m)}+k\left(d_{n}-1\right)<-\frac{(m-l) \cdot(-2)}{2(2+2 m)} \leq \varepsilon
$$

and

$$
\varepsilon \leq \frac{m+l+2}{2+2 m}=1-\frac{(m-l) \cdot 2}{2(2+2 m)}<1+k d_{n}-\frac{(m-l) \xi}{2(t+2 m)} .
$$

This finishes the proof of Theorem 3.3.

Proof of Theorem 3.4. This is very similar to the proof of Theorem 3.3. Here, we show that

$$
\begin{aligned}
u_{2 n-1} & =m 2^{n-1}+\left\lfloor t 2^{n-2}\right\rfloor, \\
u_{2 n} & =(m+l) 2^{n-1}+\left\lfloor t 2^{n-2}\right\rfloor+k\left(m 2^{n}+2\left\lfloor t 2^{n-2}\right\rfloor+1\right) .
\end{aligned}
$$

Let $m \geq 1$. Then the induction step $u_{2 n} \rightarrow u_{2 n+1}$ leads to $0 \leq(t+2 m)(2 k+1)\left(1-d_{n}\right)+l\left(t 2^{n}-2\left\lfloor t 2^{n-1}\right\rfloor\right)<(t+2 m)(2 k+1)+2 l$, 
which is obviously true. For $u_{2 n-1} \rightarrow u_{2 n}$ we end up with

$$
0 \leq((t+2 m)(2 k+1)+2 l) \varepsilon-k(t+2 m)+l \xi^{\prime \prime}<t+2 m,
$$

where $\xi^{\prime \prime}=2\left\lfloor t 2^{n-2}\right\rfloor-t 2^{n-1} \in(-2,0]$. Then

$$
\begin{aligned}
\frac{k(t+2 m)-l \xi^{\prime \prime}}{(t+2 m)(2 k+1)+2 l} & <\frac{k(1+2 m)+2 l}{(2 k+1)(1+2 m)+2 l} \\
& =\frac{1}{2}-\frac{m-l+1 / 2}{(2 k+1)(2 m+1)+2 l} \leq \varepsilon
\end{aligned}
$$

and

$$
\begin{aligned}
\varepsilon & <\frac{1}{2}+\frac{m-l+1 / 2}{(2 k+1)(2 m+1)+2 l}=\frac{(k+1)(1+2 m)}{(1+2 m)(2 k+1)+2 l} \\
& \leq \frac{(k+1)(t+2 m)-l \xi^{\prime \prime}}{(t+2 m)(2 k+1)+2 l}
\end{aligned}
$$

for all $1 \leq t<2$. This proves the statement for $m \geq 1$. Similarly, for $m \leq-2$ we get

$$
\varepsilon>\frac{k(2+2 m)+2 l}{(2+2 m)(2 k+1)+2 l} \geq \frac{k(t+2 m)-l \xi^{\prime \prime}}{(t+2 m)(2 k+1)+2 l}
$$

and

$$
\frac{(k+1)(t+2 m)-l \xi^{\prime \prime}}{(t+2 m)(2 k+1)+2 l}>\frac{(m+1)(k+1)}{(m+1)(2 k+1)+l} \geq \varepsilon .
$$

This completes the proof of Theorem 3.4.

Proof of Corollary 3.5. Put $t=w 2^{-M}=\left(d_{1} . d_{2} d_{3} \ldots\right)$. Since $0<w<2$ we deduce that $M \leq 0$ and by Proposition 4.1 and a minor inductive argument that $u_{2 n+1}-2 u_{2 n-1}=0$ if $1 \leq n \leq-M$. Therefore, it suffices to show that the sequence $v_{1}=m 2^{-M}, v_{n+1}=\left\lfloor\sqrt{2}\left(v_{n}+1 / 2\right)\right\rfloor$ satisfies $v_{2 n+1}-2 v_{2 n-1}=d_{n}$. First, let $m=\lfloor r(1+1 / \sqrt{2})\rfloor$ and set $k=0$, $l=\lfloor r / \sqrt{2}\rfloor 2^{-M}$ and $m \mapsto m 2^{-M}$ in Theorem 3.3. As for the second case $m=\lfloor r(1+\sqrt{2})\rfloor$, we use Theorem 3.4 for $k=0, l=r$ and $m \mapsto m 2^{-M}$. In both cases $a=b=\sqrt{2}$, and $\varepsilon=1 / 2$ lies in the admissible interval, such that the two cases corresponding to the parity of $n$ merge. This finishes the proof.

\section{References}

[1] J. Borwein and D. Bailey, Mathematics by Experiment, A K Peters, Natick, MA, 2004.

[2] P. Erdős and R. L. Graham, Old and New Problems and Results in Combinatorial Number Theory, Monogr. Enseign. Math. 28, Univ. Genève, 1980.

[3] A. S. Fraenkel, The bracket function and complementary sets of integers, Canad. J. Math. 21 (1969), 6-27. 
[4] R. L. Graham, D. E. Knuth and O. Patashnik, Concrete Mathematics, 2nd ed., Addison-Wesley, 1994.

[5] R. L. Graham and H. O. Pollak, Note on a nonlinear recurrence related to $\sqrt{2}$, Math. Mag. 43 (1970), 143-145.

[6] R. K. Guy, The strong law of small numbers, Amer. Math. Monthly 95 (1988), 697-712.

[7] F. K. Hwang and S. Lin, An analysis of Ford and Johnson's sorting algorithm, in: Proc. Third Annual Princeton Conf. on Inform. Sci. and Systems, J. B. Thomas, M. E. van Valkenburg and P. Weiner (eds.), Dept. of Electrical Engrg., Princeton Univ., Princeton, NJ, 1969, 292-296.

[8] S. Rabinowitz and P. Gilbert, A nonlinear recurrence yielding binary digits, Math. Mag. 64 (1991), 168-171.

[9] N. J. A. Sloane, The On-Line Encyclopedia of Integer Sequences, entries no. A091522, A091523, A091524, A091525, A100671, A100673, A001521, A004539, http: //www.research.att.com/ njas/sequences/, 1996-2006.

[10] Th. Stoll, On families of nonlinear recurrences related to digits, J. Integer Seq. 8 (2005), no. 3, article 05.3.2, 8 pp. (electronic).

Institute of Discrete Mathematics and Geometry

TU Vienna

Wiedner Hauptstrasse 8-10

1040 Wien, Austria

E-mail: stoll@dmg.tuwien.ac.at 